\title{
Reduction in proportions of dental plaque streptococci following a 5 day topical kanamycin treatment
}

\author{
W. J. LOESCHE, R. N. HockEtT AND S. A. SYED \\ Dental Research Institute, University of Michigan School of Dentistry, \\ Ann Arbor, Michigan 48104
}

\begin{abstract}
A 5 day topical kanamycin treatment of the dental gingival surfaces in institutionalized mentally retarded subjects significantly reduced the plaque mass on the tooth surfaces compared to a placebo treatment. This reduction occurred in the absence of any mechanical hygiene procedures and lasted for 4 or more weeks, which was longer than the kanamycin could have been expected to be present in the mouth. This suggested that the kanamycin had caksed an alteration of the plaque flora which should be discernible by quantitative culturing procedures. Plaque was removed and cultured, using an anaerobiic serial dilution procedure, before and after kanamycin or placebo treatments. The reduction in plaque weight was associated with reduced levels of streptococci in the plaque. This could be demonstrated by intergroup comparison of the placebo - and kanamycin treated subjects, and by intragroup comparisons of the kanamycin treated subjects prior to treatment and at 4 and 8 weeks after treatment. The streptococci species accounted for $30 \%$ of the plaque colony forming units (CFU) prior to kanamycin treatment. At 4 weeks after treatment, the streptococci had decreased to $3 \%$ of the CFU and at 8 weeks after treatment, the streptococci had increased slightly to account for $14 \%$ of the CFU. The streptococci appeared to be Streptocaccus sanguis and Streptococcus mitis. The observations indicate that the topical kanamycin was effective in reducing supragingival plaque because of its ability to decrease the proportions of streptococci in the plaque.
\end{abstract}

(Accepted for publication May 5, 1976)

\section{Introduction}

Periodontal disease is a chronic inflammatory process in the supporting structures of the teeth which over a period of time, may result in loss of the teeth. The inflammation appears to be in response to the bacteria resident on the dento-gingival surfaces, in that germfree mice show significantly less gingival inflammation than conventional mice (Gibbons \& Socransky 1966). The bacteria do not appear to invade the tissue, but rather produce substances which presumably enter the tissue and initiate the inflammatory response (Socransky 1970). It is not known whether specific bacteria in the plaque are responsible for the inflammation, or whether increased plaque weight, by producing more irritants, leads to a more pronounced host response. Traditional dental therapy assumes that increased plaqne weight per se is the determinant of periodontal pathology, and treatment consists of mechanical debridement in order to maintain low levels of plaque (Glickman 1971). In recent years, evidence has appeared which indicates that 
specific plaque bacteria may be etiologically responsible for certain types of periodontal disease in animals. Actinomyces viscosus (Jordan \& Keyes 1964, Jordan et al. 1972) and Actinomyces naeslundii (Socransky et al. 1970), isolated from both human and animal sources, cause extensive alveolar bone loss in germfree and conventional rodents. Several unidentified Bacillus species are responsible for a transmissible periodontal syndrome in the rice rat (Dick et al. 1967) Streptococcus salivarits (Kelstrup \& Gibbons 1970) cause alveolar bone loss in germfree rodents. If these or yet unidentified organisms contribute to human periodontal disease, then dental therapy should be directed toward their elimination or suppression in the plaque flora. This might be accomplished by short term intensive chemotherapy of the dental gingival surfaces either alone, or in conjunction with mechanical debridement.

A 5-day treatment with topical kanamycin involving institutionalized mentally retarded subjects, significantly reduced the plaque weight as compared to a placebo treatment (Loesche et al. 1971). This was observed clinically during the period of kanamycin application and was documented 4 weeks later when the plaque was quantitativeley removed and weighed. This reduction in plaque weight was monitored longer than the kanamycin could have been expected to persist at antimicrobial levels in the mouth. This suggested that the kanamycin had caused an alteration of the plaque flora, which because of its profound effect on plaque weight should be discernible by quantitatively culturing procedures. This paper reports the results of this quantitative cultural study.

\section{Materials and Methods}

Subjects. Eleven mentally retarded subjects, aged 11-22 years, who were residents of a single dormitory at the Plymouth State Home and Training School, Northville Michigan, were treated with either kanamycin paste (6 subjects) or placebo paste (5 subjects). The individuals, on initial examination, exhibited advanced gingivitis i.e. a gingivitis score of $2.54 \pm 0.44$ as determined by a modification of the gingivitis index of Löe and Silness (Loesche \& Nafe 1973) and had supra-gingival plaque accumulations of about $4 \mathrm{mg}$ wet weight per reference tooth. Tooth brushing was suspended during the experimental period so that the effect of this mechanical debriding procedure on the plaque flora was eliminated, All subjects consumed the same nutritionally adequate institutional diet which was high in starch. Sucrose was rarely consumed between meals.

Treatment schedule. The treatment schedule and number of patients treated at one time revolved around the bacteriological aspects of the study. A four week interval was necessary between plaque samplings so that the bacterial isolates could be partially characterized. The subjects were treated in groups of 3, 4, and 4 subjects.

At the first visit (week 0) all teeth of all patients were mechanically scaled and polished to insure low initial plaque measurements. The subjects were placed on a no mechanical hygiene regimen for the next 12 weeks. After 4, 8 and 12 weeks the supragingival and marginal plaque on 4 representative teeth was "quantitatively removed" and immediately placed into a reduced transport fluid (RTF) (Loesche et al. 1972, Syed \& Loesche 1972). After the 4 week plaque collection, the patients were separated into placebo and kanamycin groups and given either Orabase Emollient (Placebo) (Davis, Rose Hoyt Pharmaceutical Co., Needham, Mass.) or Orabase with $5 \%$ kanamycin (Kantrex ${ }^{0}$, Bristol Laboratory, Syracuse, N. Y.). The kanamycin or placebo pastes were applied with a cotton swab in 
the morning, afternoon and evening of 5 consecutive days to the buccal labial surfaces of all teeth by a member of the investigating team. At weeks 8 and 12 the subjects were reexamined and all plaque and bacteriology procedures were repeated. Thus 4 week old plaque samples were obtained from each subject at week 4 ( 4 weeks after the scaling and polishing and just prior to kanamycin or placebo treatment); at week 8 (8 weeks after the scaling and polishing and $3^{1 / 3}$ weeks after cessation of kanamycin or placebo treatment); at week 12 ( 12 weeks after the prophylaxis and $71 / 3$ weeks after cessation of kanamycin ar placebo treatment).

Bacteriological Procedures. Supragingival and marginal plaque from the mesial buccal, buccal, and distal buccal surfaces was "quantitatively removed" with periodontal scalers from the maxillary left second premolar, maxillary left first molar, mandibular right second premolar, and mandibular right first molar. The plaque was immediately placed in $1 \mathrm{ml}$ of RTF contained in a shell vial. The vials were stoppered and placed into a Brewer jar whose atmosphere was made anaerobic by a Gas Pak (Bioquest) and returned to the laboratory within 2 to 3 hours.

Sample dispersion and plating. The vial containing the plaque was introduced into a plastic anaerobic chamber (Aranki et al. 1969) containing an atmosphere of $85 \%$ $\mathrm{Ne}_{2}, 10 \% \mathrm{~Hz}$ and $5 \% \mathrm{CO}$. All subsequent manipulations and incubations were performed in the chamber. The aluminum foil around the vial and cork stopper were removed. The vial and its contents were added to $100 \mathrm{ml}$ of RTF contained in a Waring blender. The plaque was dispersed by mixing at the highest setting of the blender for 2 minutes. Aliquots from the blender dilution were: 1) stained with buffered formalin, gentian violet dye (Aranki et al. 1969) and a microscopic count was obtained using a Petroff Hauser counting chamber and 2) carried through a serial 10 fold dilution in RTF. A membrane filter technique was used to harvest the bacteria from the serial dilution tubes (Loesche et al. 1972). One ml aliquots of the appropriate dilution were filtered using $0.22 \mu \mathrm{m}$ membrane filters (Millipore Corp., Bedford, Mass.). The filters containing the impinged bacterial cells were placed on the surface of MM10 medium with and without $0.05 \%$ kanamycin and on mitis salivarius agar. The MM10 medium is a $0.2 \%$ trypticase, $0.5 \%$ yeast extract, $3 \%$ sheep blood medium which is poised with $0.01 \%$ dithiothreitol and supplemented with the known nutrient requirements of some members of the oral flora (Loesche et al. 1972). For each plaque sample, duplicate aliquots of 3 tenfold dilutions were incubated anaerobically and 2 tenfold dilutions were incubated aerobically.

Parial characterization of isolates. The MM10 plates were incubated for 5 to 7 days. The number of colonies on the plates was counted with the aid of a dissecting microscope. One anaerobic MM10 plate from each plaque sample, containing from 20 to 100 well separated colonies, was selected for further study. The plate was kept within the chamber and each colony was streaked onto MM10 agar for a purity check. This was necessary because abont 10 to $15 \%$ of the primary colonies were mixtures of 2 or more bacterial types. Isolates which appeared pure by Gram stain and darkfield examination were subcultured to a characterization broth (Loesche et al. 1972). After growth in this medium, the following tests were performed: terminal $\mathrm{pH}$ in glucose broth, $\mathbb{H}_{2} \mathrm{~S}$ production determined by blackening of lead acetate strips, nitrate reduction, gelatin liquefaction and indole production following methods described by the 
Society of American Microbiologists (1957). Growth from the characterization broth was streaked on MM10 agar plates and incubated aerobically to determine whether the isolates were facultative organisms. Representative strains were subcultured in peptone, yeast extract, glucose broth (PYG) and their acid end products were determined by gas-liquid chromatographic procedures described in the Virginia Polytechnic Institute anaerobic manual. Approximately 250 isolates were characterized in this fashion every 4 weeks giving a total of about 1600 isolates for the 36 week period of study.

\section{Results}

Pooled plaque from 4 representative teeth in 6 kanamycin treated and 5 placebo treated subjects was cultured at 4,8 and 12 weeks. The percent recovery of organisms for each sample was calculated by dividing the anaerobic colony forming unit (CFU) count by the microscopic count. These values aver- aged from 25 to $58 \%$ for each time interval and are shown in Table 1 . The anaerobic count was higher than the aerobic count for each sample. The anaerobic to aerobic CFU ratio was calculated for each sample and the average values for each time interval varied from 2.6 to 12 (Table 1 ). The value of 12 was abtained from the kanamycin treated subjects at week 8 , and was due to a decrease in the streptococci count (Table 2).

All colonies from one dilution plate per sample were subcultured. Ninety percent of the isolates grew and were partially characterized. There was no discernible difference between the flora of the two treatment groups at the week 4 sampling period prior to the kanamycin or placebo treatment. When the flora was re-examined at week 8 , the gram positive cocci had significantly decreased in the kanamycin-treated group from 32 to $8 \%$ of the CFU, $p=0.01$ (Student $t$ test). The percentage of strongly acidogenic organisms had decreased from 34 to $11 \%$, $\mathrm{p}<0.05$ ( $t$ test), whereas the nonacidogenic flora had increased from 28 to $64 \%, \mathrm{p}<$

Table 1

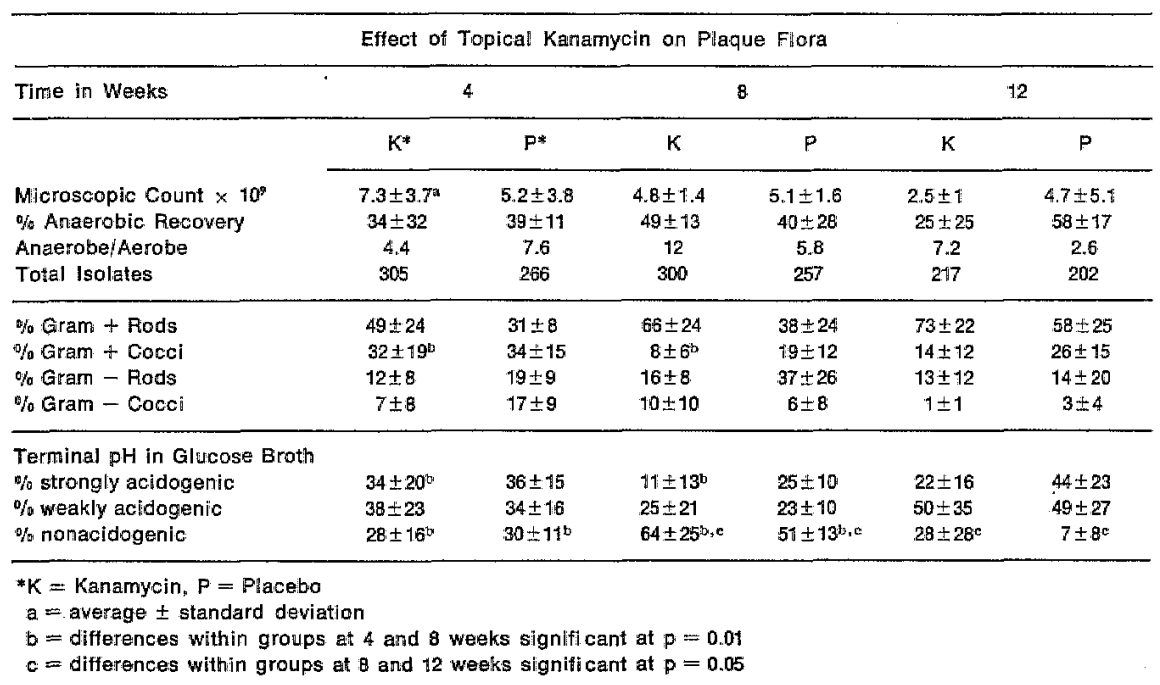


Table 2

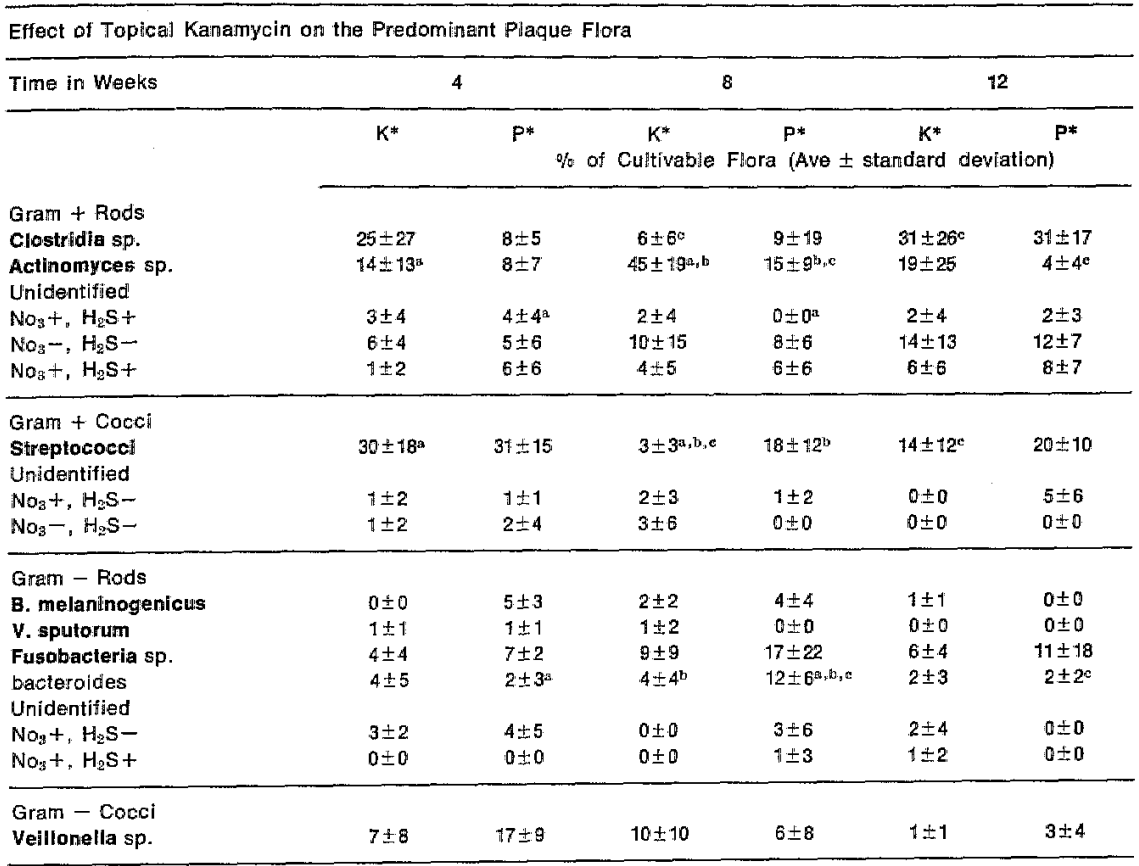

${ }^{*} \mathrm{~K}=$ Kanamycin, $\mathrm{P}=$ Placebo

$a=$ difference within groups at 4 and 8 weeks is significant $p<0.0 t$

$b=$ difference between $K$ and $P$ at weok $B$ is significant $p<0.02$

$c=$ difference within groups at $B$ and 12 weeks is significant $p<0.05$

0.05 . The placebo-treated group also showed a significant $(p<0.05)$ increase in nonacidogenic organisms. The kanamycin-treated group showed a nonsignificant increase in the percentage of gram positive rods and the placebo-treated group exhibited a nonsignificant increase in the percentage of gram negative rods. The intergroup differences were not significant, even though the placebo-treated group had twice the percentage of gram positive cocci and acidogenic organisms. At week 12, the percentage of nonacidogenic organisms had dropped significantly in both groups. There were nonsignificant increases in the weakly acid- ogenic organisms i.e. terminal $\mathrm{pH}$ in glucose broth was between 5.5 and 6.5 . The percentage of gram positive cocci and acidogenic organisms was still reduced in the kanamycin-treated group relative to the placebo-treated group.

The isolates were separated into genera and unidentified groups on the basis of gram stain morphology and the limited number of biochemical tests used. The gram positive rods, accounted for 31 to $72 \%$ of the isolates (Table 1). These organisms were separated into 5 groups: anaerobic, sporulating rods identified as Clostridium species; branching or filamentous rods considered to 
be Actinomyces species; and three groups that were separated according to their ability to reduce nitrate and produce $\mathrm{H}_{2} \mathrm{~S}$ (Table 2).

The clostridia were not uniformly isolated from all subjects at all times. They were reduced from $25 \%$ to $6 \%$ of the CFU at week 8 , following kanamycin therapy, but significantly increased to $31 \%$ in the 12 week sample (Table 2). These organisms included strains of Clostridium sporogenes, Clostridium hastiforme, and Clostridium subterminale. Some isolates were capable of aerobic growth and were found to be Clostridium histolyticum (Loesche et al. 1972, Loesche et al. 1974).

Actinomyces isolates were nitrate positive, and gelatin, indole and HeS negative. They increased significantly in the kanamycin group at week 8 and then decreased at week 12. At week 8 , they accounted for $45 \%$ of the plaque isolates, which was significantly higher than their proportion in the placebo group.

Gram positive cocci accounted for 8 to $34 \%$ of the isolates at the various times of sampling. They could be divided into a large group containing Streptococcus species and two smaller groups separated according to nitrate reduction and $\mathrm{H}_{2} \mathrm{~S}$ production. The streptococcal isolates did not exhibit the colonial morphology of Streptococcus mutans and Streptococcus salivarius when plated on Mitis Salivarius agar. These isolates were considered to be Streptococcus sanguis and Streptococcus mitis and were not further differentiated. At week 8, in the kanamycin group they decreased to only $3 \%$ of the isolates. This reduction was significant when compared to the pretreatment value of $30 \%$ and the placebo value of $18 \%$ (Table 2). At week 12 , in the kanamycin group, the streptococei increased significantly so as to account for $14 \%$ of the flora. In the placebo group, the streptococci decreased nonsignificantly at week 8 to $18 \%$, and remained at this level at week 12 . The other groups of gram positive cocci accounted for less than $5 \%$ of the isolates and did not seem to be influenced by either the kanamycin or the placebo treatment.

Gram negative rods accounted for 12 to $37 \%$ of the isolates. Bacteroides melaninogenicus ranged from 0 to $5 \%$ of the isolates. Vibrio (Campylobacter) sputonum was a minor organism and was seen only occasionally. Strains were considered to be bacteroides if they fermented glucose and were nitrate and $\mathrm{H}_{2} \mathrm{~S}$ negative. Representative isolates formed succinate in PYG broth. The bacteroides represented 1 to $12 \%$ of the isolates and significantly increased in the placebo group at week 8 (Table 2). Fusobacterium species were more numerous, i.e. 4 to $17 \%$ of the CFU. These isolates were nitrate negative, indole variable, gelatin negative, $\mathrm{HzS}$ positive, and non- to weakly acidogenic in glucose broth. The kanamycin or placebo treatment was associated with a nonsignificant increase in the percentage of these organisms at week 8 . The unidentified gram negative rods which would include Leptotrichia, Eikenella and Capnocytophagia strains made minimal contributions to the flora and did not seem to be affected by the treatment pastes.

One to $17 \%$ of the isolates were nitrate positive, nonacidogenic gram negative anaerobic cocci and were considered to be Vellonella species. Their percentages in plaque were not altered by the treatment pastes.

The absolute numbers of streptococci and actinomyces strains in the plaques at weeks 4,8 and 12 were calculated from the microscopic count (Table 1). At week 4 , slightly higher numbers of streptococci and actinomyces were present in the plaques taken from the kanamycin subjects (Table 3) and the streptococci to actinomyees ratio ranged from 2 to 4 . In the 8 week kanamycin plaques, a 10 fold decrease in streptococci 
Table 3

Effect of Kanamycir or Placebo Paste on Absolute Numbers of Streptococel and Actinomyces Species in Supragirgival Plague

\begin{tabular}{lcccccc}
\hline Time in Weeks & & & 8 & & 12 \\
\hline Treatment Group & $K^{\mathrm{a}}$ & $\mathrm{p}$ & $\mathrm{K}$ & $\mathrm{P}$ & $\mathrm{K}$ & $\mathrm{P}$ \\
\hline streptococci sp. & $7.4^{\mathrm{b}}$ & 6.3 & 0.7 & 3.7 & 0.9 & 5.5 \\
actinomyces sp. & 3.5 & 1.6 & 10.6 & 3.1 & 1.2 & 1.1 \\
\hline $\begin{array}{l}\text { Ratio } \\
\text { Streptococei to } \\
\text { Actinomyces }\end{array}$ & 2.1 & 3.9 & 0.07 & 1.2 & 0.75 & 5.0 \\
\hline
\end{tabular}

$a=K$ is kanamycin; $P$ is placebo

b All values are $\times 10^{\circ}$. These values are calculated from microscopic count, \% anaerobic recovery and $\%$ Streplococci or \%o Actinomyces values given in Tabies 1 and 2

and a 3 fold increase in actinomyces caused a sharp drop in this ratio. No such drop occurred in the placebo group. In the 12 week kanamycin plaques, the streptococci were still reduced, but now the actinomyces levels were lower, so that the streptococci to actinomyces ratio approached the before treatment ratio seen at week 4 . The placebo plaques did not exhibit wide fluctuations in this ratio.

Approximately 1 to $5 \%$ of the cultivable flora in both the kanamycin and placebo treated subjects was resistant to $0.05 \%$ kanamycin at the start of the clinical trial. No increase in numbers of plaque organisms resistant to $0.05 \%$ kanamycin was observed at any subsequent time interval.

\section{Discussion}

Topical kanamycin caused a reduction in supragingival plaque mass of at least 4 weeks duration. When the plaque mass on the 6 index teeth decreased from 4.4 to $2.5 \mathrm{mg}$ dry wt, (Loesche et al. 1971) the streptococci decreased on the other test teeth from $30 \%$ to $3 \%$ of the CFU. When the plaque weight increased slightly at week 12 , the streptococci increased to $14 \%$ of the CFU.
No other group of arganisms exhibited a similar positive association with the plaque weight in the kanamycin-treated subjects.

The actinomyces species exhibited a proportional increase in the kanamycin treated group at week 8 . This proportional increase not only reflected the deletion of the streptocoeci from the sample, but also a real increase in the numbers of actinomyces in the plaque (Table 3). In vitro studies have shown these organisms to be more resistant to kanamycin than the various Streptococcus species (S. Damle, personal communication). Members of this genus, especially $A$. viscosus isolated from mentally retarded individuals (Jordan et al. 1972) and A. naeslundii isolated from human plaque (Socransky et al. 1970 ) will cause periodontal destruction in animal models. The prominence and persistance of these organisms in the week 8 plaque might account for the inability of the kanamycin treatment to restore gingival health (Loesche et al. 1971). Thus drugs with a broader spectrum of in vivo activity against plaque bacteria might have been more effective.

The proportions of the nonpigmented bacteroides group increased significantly in the placebo group at week 8 for reasons un. 
known. These organisms at other time periods comprised less than $5 \%$ of the CFU. Thus the possibility of a proportional increase of these organisms in the placebo group contributing to the intergroup differences in plaque weight at week 8 should be recognized.

Our characterization of the isolates was not adequate to determine whether plaque levels of a single species, such as $A$. viscosus or $\mathrm{Cl}$. histolyticum, had changed. Thus the possibility exists that within groups such as the Clostridia, Actinomyces, etc., a shift of species could have occurred as a result of kanamycin treatment.

These data implicate the streptococci as the organisms which contribute in a substantial manner to plaque weight in these institutionalized subjects. There are several ways in which streptococci contribute to plaque formation and/or accumulation on animal and human teeth. Streptococcus mutans forms massive amounts of plaque in animals fed diets high in sucrose, by synthesizing extracellular glucans which adhere to the tooth surfaces (Gibbons \& Nygaard 1968, Guggenheim 1970). S. mutans was not found in the present plaque samples, as determined by colonial morphology on mitis salivarius agar, so that a reduction in the level of this species cannot explain the present observations. The streptococci found in our samples appeared to be $S$. sanguis and S. mitis. S. sanguis and S. mitis are adsorbed to the teeth from saliva and are among the first colonizers of the tooth surface (van Houte et al. 1970, van Houte et al. 1971). Cultural and microscopic studies of young plaque have revealed the presence of high levels of streptococci (Carlsson 1965, Theilade \& Theilade 1970). Seanning electronphotomicrographs taken of teeth at hourly intervals following a mechanical prophylaxis, show the deposition and accumulation of clumps of bacteria which resenble streptococci (Saxton 1973). Transmission elec- tron-photomicrographs of plaque on the tooth surface show coccal organisms predominating at the enamel plaque interface (Frank \& Houver 1970, Schroeder \& Hirzel 1969).

These studies suggest that streptococci initiate most plaques formed on teeth. If, during the time of tooth colonization, the level of species such as $S$. sanguis in the saliva was lower than the $10^{3}$ organisms $/ \mathrm{ml}$ estimated to be necessary for attachment (van Houte \& Green 1974), then it is possible that other organisms with lower affinities for the tooth surface, but with greater resistance to the kanamycin could attach. If the time span in which the streptococci were suppressed was sufficiently long i.e. 5 days, then most, if not all tooth surfaces could be colonized by the organisms with the lower affinity, which in the present investigation appeared to be the various Actinomyces species. The actinomyces dominated plaque would persist as long as the streptococci were not permitted access to the tooth surface. This period would extend for the time in which the plaque was not disturbed, which in our experiment was the 4 week interval of no brushing. Thus, it is possible that the no brushing regime, instituted to remove the variable of unequal brushing efficacy on the part of the subjects, may have potentiated the effect of the kanamycin treatment. When plaque was removed at week 8 to be cultured, the exposed tooth surfaces would be bathed by saliva containing presumably normal levels of streptos cocci. Hence streptococci would be more likely to initiate plaque formation at this time, and indeed when the plaque was sampled at week 12, both the streptococcal levels and weight had increased. This suggested that at any time the plaque was removed, kanamycin treatment should be given to minimize colonization by the streptococci. This retreatment approach was tested in a separate clinical study in which 
topical kanamycin was applied for 3 to 5 days every fifth week to Down's syndrome subjects. After 45 weeks of no brushing, plaque levels were about half the pretreatment amounts and a clinically acceptable gingival health was obtained in some subjects (Loesche \& Nafe 1973).

These bacteriological observations indicate that streptococci contribute to plaque mass. When these organisms were discriminated against by short term kanamycin therapy, the Actinomyces were able to dominate in the plaque samples. This plaque did not possess the mass of the previous plaque and was associated with a slight improvement in the gingivitis score of the patient (Loesche et al. 1971).

\section{Acknowledgements}

The assistance of Mrs. Janice Stoll and Mrs. Joan Rowan in the bacteriological aspects of this study is gratefully acknowledged. Dr. Don Nafe and his staff at the Plymouth State Home and Training School, Northville, Michigan provided assistance in the clinical aspects of this study. This work was supported by grants from the National Institute of Dental Research No. DE-03011 and No. DE-02731.

\section{References}

Aranki, A., Syed, S. A., Kenney, E. B. \& Freter, R. 1969. Isolation of anaerobic bacteria from human gingiva and mouse cecum by means of a simplified glove box procedure. Appld. Microbiol. 17: 568-576.

Carlsson, J. 1965. Zooglea-forming streptococci resembling Streptococcus sangius isolated from dental plaque in man. Odont. Revy 16: 348-358.

Dick, D. S., Shaw, J. \& Socransky, S. S. 1967. Further studies on the microbial agent or agents responsible for the periodontal syndrome in the rice rat, Arch. Oral Biol. 13: 215-228.

Frank, R. M. \& Houver, G. 1970. An ultra- struetural study of human supragingival dental plaque formation. Ch. in Dental Plaque p. 85-108; W. D. McHugh ed. E. S. Living stone Ltd. Edinburgh.

Gibbons, R. J. \& Nygaard, M. 1968. Synthesis of insoluble dextran and its significance in the formation of gelatinous deposits by plaque-forming streptococci. Arch. Oral Biol. 13: $1249-62$.

Gibbons, R. J. \& Socransky, S. S. 1966. Enhancement of alveolar bone loss in gnotobiotic mice harboring human gingival bacteria. Arch. Oral Biol. 11: 847-848.

Gibbons, R. J. \& van Houte, J. 1973, On the formation of dental plaques. J. Periodontol. 44: $347-360$.

Glickman, I. 1971. Periodontal Disease. N. Engl. J. Med. 284: 1071-7.

Guggenheim, B. 1970. Extracellular polysaccharides and microbial plaque. Int. Dent. J. 20: $657-678$.

Irving, J. T., Newman, M. G., Socransky, S. S. \&. Heeley, J. 1975. Histological changes in experimental periodontal disease in rats monoinfected with a gram-negative organism. Arch. Oral Biol. 19: 219-222.

Jordan, H. V. \& Keyes, P. H. 1964. Aerobic, granu-positive, filamentous bacteria as etiologic agents of experimental periodontal disease in hamsters. Arch. Oral Biol. 9: 401414.

Jordan, H. V., Keyes, P. H. \& Bellock, S. 1972. Periodontal lesions in hamsters and gnotobiotic rats infected with actinomyces of human origin. J. Periodontal Res. 7: 21-28.

Kelstrup, J. \& Gibbons, R. J. 1970. Induction of dental caries and alveolar bone loss by a human isolate resembling Streptococcus saliparius. Caries Res. 4: 360-377.

Löe, H. \& Rindom Schiött, C. 1970. "The effect of mouthrinses and topical application of chlorhexidine on the development of dental plaque and gingivitis in man. J. Periodontal Res. 5: 79-83.

Loesche, W. J. \& Nafe, D. 1973. Reduction of supragingival plaque accumulations in institutionalized Down's Syndrome patients by periodic treatment with topical kanamycin. Arch. Oral Biol. 18: 1131-1143.

Loesche, W. J., Hockett, R. N. \& Syed, S. A. 1972. The predominant cultivable flora of tooth surface plaque removed from institutionalized subjects. Arch. Oral Biol. 17: 1311-1326.

Loesche, W. J., Green E., Kenmey, E. B. \& Nafe, D. 1971. Effect of topical kanamycin 
sulfate ofl plaque accumulation. $J$. Amer Dent. Assoc. 83: 1063-1069.

Loesche, W. J., Paunio, K. U., Woolfolk, M. P. \& Hockett, R. N. 1974. Collagenolytic activity of dental plaque associated with periodontal pathology. Infect. and Immunity 9: $329-336$

Newman, M. G., Socransky, S. S. \& Listgarten, M. A. 1974. Relationship of microorganisms to the etiology of periodontosis. Intern'I Assoc. Dent. Res., Abstract \# 324, Atlanta.

Ramfjord, S. P. 1959. Indices for prevalence and incidence of periodontal disease. J. Periodontol. 30: 51-58.

Saxton, C. A. 1973. Scanning ellectron microscope study of the formation of dental plaque. Caries Res. 7: 102-119.

Schroeder, H. H. \& Hirzel, H. D. 1969. A method of studying dental plaque morphology. Helvet. Odont. Acta. 13: 22-27.

Society of American Bacteriologists. Manual of microbiological methods. McGraw-Hill, New York. 1957.

Socransky, S. S. 1970. Relationship of bacteria to the etiology of periodontal disease. $J$. Dent. Res. 49: 203-222.

Sacransky, S. S., Hubersak, C. \& Propas, D. 1970. Induction of periodontal destruction in gnotobiotic tats by a human oral strain of Actinomyces naeslundii. Arch. Oral Biol. 15: 993-995.
Syed, S. \& Loesche, W. J. 1972. Survival of human dental plaque flora in various transport media. Appl. Microbiol. 24: 638-644.

Theilade, E. \& Theilade, J. 1970. Bacteriological and ultrastructural studies of developing dental plaque. Ch. in Dental Plaque (edited by McHugh, W. D.) pp. $27-40$ (Livingstone, Edinburgh).

van Houte, J. \& Green, D. B. 1974. Relationship between the concentration of bacteria in saliva and the colonization of teeth in humans. Infect. and Immun. 9: 624-630.

van Houte, J., Gibbons, R. J. \& Banghart, S. B. 1970. Adherence as a determinant of the presence of Strepiococcus salivarius and Streptococcus sanguis on the human tooth surface. Arch. Oral Biol. 15: 1025-1034.

van Honte, J., Gibbons, R. J. \& Pulkkinen, A. J. 1971. Adherence as an ecological determinant for streptococci in the human mouth. Arch. Oral Biol. 16: 1131-1141.

Virginia Polytechnic Institute Laboratory. 1970. Outline of clinical methods in anaerobic bacteriology. The Anaerobe Laboratory, Virginia Polytechnic Institute, Blacksburg, VA.

Address:

School of Dentistry

University of Michigan

Ann Arbor, Michigan 48104 
This document is a scanned copy of a printed document. No warranty is given about the accuracy of the copy. Users should refer to the original published version of the material. 